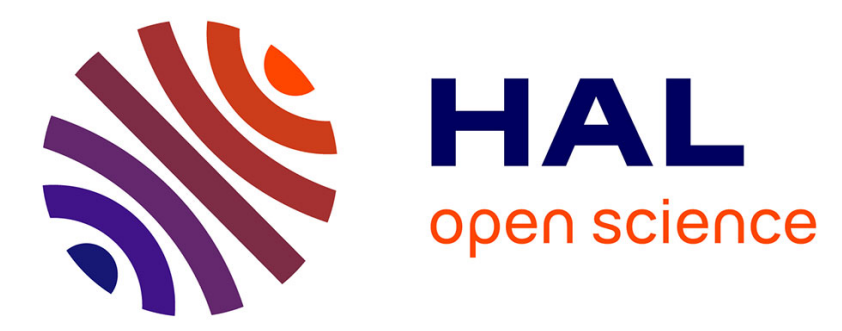

\title{
11B MAS NMR study of Ga1-xFexBO3 mixed crystals
}

K. Seleznyova, N.A. Sergeev, M. Olszewski, P. Stępień, S.V. Yagupov, M.B.

Strugatsky, Janis Kliava

\section{To cite this version:}

K. Seleznyova, N.A. Sergeev, M. Olszewski, P. Stępień, S.V. Yagupov, et al.. 11B MAS NMR study of Ga1-xFexBO3 mixed crystals. Solid State Nuclear Magnetic Resonance, 2015, 70, pp.3842. 10.1016/j.ssnmr.2015.06.001 . hal-01222354

\section{HAL Id: hal-01222354 \\ https://hal.science/hal-01222354}

Submitted on 29 Oct 2015

HAL is a multi-disciplinary open access archive for the deposit and dissemination of scientific research documents, whether they are published or not. The documents may come from teaching and research institutions in France or abroad, or from public or private research centers.
L'archive ouverte pluridisciplinaire $\mathbf{H A L}$, est destinée au dépôt et à la diffusion de documents scientifiques de niveau recherche, publiés ou non, émanant des établissements d'enseignement et de recherche français ou étrangers, des laboratoires publics ou privés.

\section{다(1)(2)}

Distributed under a Creative Commons Attribution - ShareAlikel 4.0 International 


\title{
${ }^{11}$ B MAS NMR study of $\mathrm{Ga}_{1-x} \mathrm{Fe}_{x} \mathrm{BO}_{3}$ mixed crystals
}

\author{
K. Seleznyova a,b N.A. Sergeev ${ }^{\text {c,* }}{ }^{\text {, M. Olszewski }}{ }^{\text {c }}$ P. Stępień ${ }^{c}$, S.V. Yagupov ${ }^{\text {, }}$ \\ M.B. Strugatsky ${ }^{a}$, J. Kliava ${ }^{b}$ \\ a Faculty of Physics, Taurida National V.I. Vernadsky University, 95-007 Simferopol, Crimea, Ukraine \\ b LOMA, UMR 5798 Université de Bordeaux-CNRS, 33405 Talence cedex, France \\ ${ }^{\mathrm{c}}$ Institute of Physics, University of Szczecin, 15 Wielkopolska Str., 70-451 Szczecin, Poland
}

Keywords:

borate compounds

${ }^{11}$ B MAS NMR

Local structure

Local disorder.

\section{A B S T R A C T:}

Mixed iron-gallium borate crystals $\mathrm{Ga}_{1-x} \mathrm{Fe}_{x} \mathrm{BO}_{3}$ have been studied by Magic Angle Spinning (MAS) NMR of ${ }^{11} \mathrm{~B}$ isotope. Experimental MAS NMR spectra have been computer simulated using a laboratory-developed code. The quadrupole parameters and isotropic chemical shift for ${ }^{11} \mathrm{~B}$ are consistent with threefold-coordination of boron atoms. A detailed fitting to the experimental NMR spectra reveals the existence of a certain local disorder in $\mathrm{Ga}_{1-x} \mathrm{Fe}_{x} \mathrm{BO}_{3}$ crystals.

\section{Introduction}

Crystals of borate family with chemical formula $\mathrm{M}^{3+} \mathrm{BO}_{3}$, where $\mathrm{M}^{3+}=\mathrm{Ti}, \mathrm{V}, \mathrm{Cr}, \mathrm{Fe}, \mathrm{Ga}$, etc., are crystallized in $D_{3 d}^{6}$ space group and have a calcite structure with two formula units per unit cell. For different $\mathrm{M}^{3+}$ ions, these crystals possess very different electronic, magnetic and optical properties [1]. For instance, vanadium borate, $\mathrm{VBO}_{3}$ is semiconducting and ferromagnetic with $T_{\mathrm{C}}=32.5 \mathrm{~K}[1]$ while iron borate, $\mathrm{FeBO}_{3}$ is insulating and canted antiferromagnetic below $T_{\mathrm{N}}=348 \mathrm{~K}[2]$. Besides, iron borate combines room-temperature magnetic ordering with considerable transparency in visible spectral range; therefore, it is a good candidate for magneto-optical applications [3-5]. $\mathrm{FeBO}_{3}$ single crystals are also known for their extraordinary magnetic and acoustic resonance properties [6].

Solid solutions of $\mathrm{M}^{3+} \mathrm{BO}_{3}$ crystals with different $\mathrm{M}^{3+}$ ions are promising materials for solid-state lasers and acousto-optic devices due to their extraordinary optical, mechanical and thermal properties [1-5]. These properties substantially depend on chemical composition and structural and compositional ordering of the crystals. A certain degree of disorder, expected to be present in such crystals, manifests itself in inhomogeneous distribution of local electric fields and can be suitably studied by NMR [7]. In the case of NMR of quadrupolar nuclei, structural information is contained in the chemical shift $\delta$ and quadrupole coupling parameters

\footnotetext{
* Corresponding author.

E-mail address: sergeev@wmf.univ.szczecin.pl (N.A. Sergeev).
}

$[8,9]$ which can be extracted from experimental spectra by computer simulations. Several NMR simulation programs are available, e.g., GAMMA, SIMPSON, Winfit, STARS, Wsolids, QUASAR, QuadFit and DMFit [10-18]. Unfortunately, most of these programs either do not take into account distributions of NMR parameters caused by local disorder, or, in the best case, employ insufficiently general distribution forms [19].

In the present work, the Magic Angle Spinning (MAS) NMR of ${ }^{11} \mathrm{~B}$ has been used for the first time to study iron-doped gallium borates, $\mathrm{Ga}_{1-x} \mathrm{Fe}_{x} \mathrm{BO}_{3}(x=0,0.01,0.02)$. Experimental MAS NMR spectra have been computer simulated using a laboratory-developed code explicitly taking into account the distribution of the quadrupole parameters in the most general form, the so-called Maurer's distribution [20]. The results of our EPR study of these crystals have been published elsewhere [21].

\section{Experimental details}

\subsection{Sample preparation}

$\mathrm{Ga}_{1-x} \mathrm{Fe}_{x} \mathrm{BO}_{3}$ single crystals were synthesized by solution-in-melt crystallization [22] carried out in $\mathrm{Ga}_{2} \mathrm{O}_{3}-\mathrm{Fe}_{2} \mathrm{O}_{3}-\mathrm{B}_{2} \mathrm{O}_{3}-\mathrm{PbO}-\mathrm{PbF}_{2}$ system. Previous probe method and differential thermal analysis have allowed determining charge component ratios and corresponding temperature modes optimal for the crystal synthesis. In particular, significant adjustment of the temperature mode was necessary in order to achieve good crystallization results for different $x$ values. 
The contents of various chemical elements in the synthesized crystals were determined by X-ray fluorescence analysis (XRF) and energy-dispersive spectroscopy [22]. XRF showed significant differences between gallium and iron concentrations in the charge and in the synthesized crystals. Besides, considerable concentration variations were observed for crystals grown in the same crucible; for instance, with an initial iron concentration of $1 \%$ in the charge, the spread of iron concentrations in the crystals was from $0.2 \%$ to $4.0 \%$.

\subsection{Experimental techniques and equipment}

The ${ }^{11} \mathrm{~B}$ MAS NMR spectra of $\mathrm{Ga}_{1-x} \mathrm{Fe}_{x} \mathrm{BO}_{3}$ ground to powders sufficiently fine to avoid preferential crystallite orientations, were measured at $128.384 \mathrm{MHz}$ frequency in $9.4 \mathrm{~T}$ magnetic field using a Bruker Avance-400 NMR spectrometer. A $4 \mathrm{~mm}$ in diameter zirconia $\left(\mathrm{ZrO}_{2}\right)$ rotor cell equipped with $\mathrm{BN}$ stator filled with powdered sample was spinning under magic angle at $10 \mathrm{kHz}$ frequency. A $90^{\circ}-\tau-180^{\circ}-\tau$-Acq Hahn two pulse echo sequence with echo delay $\tau$ has been used to record NMR signals from ${ }^{11} \mathrm{~B}$ nuclei $[23-26]$. ${ }^{11} \mathrm{~B}$ nucleus has $I=3 / 2$ nuclear spin, and for selective excitation of the central transition $\pm 1 / 2 \leftrightarrow \mp 1 / 2$ the optimal pulse duration equals to that of a non-selective $\pi / 2$ pulse divided by $I+1 / 2=2[8,9]$. In our experiments, the duration of the first radiofrequency (RF) pulse in the Hahn two pulse echo sequence was $t_{\mathrm{i}}=1.75 \mu \mathrm{s}$. The recycle delay between acquisitions was $0.5 \mathrm{~s}$, and a total of 1024 acquisitions was sufficient enough to resolve the characteristic borate spectral features for the polycrystalline samples.

The ${ }^{11} \mathrm{~B}$ NMR spectra were computer simulated using a laboratory-developed code. For the sake of comparison, the DMFit program [18] was also used in some simulations.

\section{Results and discussion}

\subsection{Experimental results and "standard" computer treatment}

Figure 1 shows ${ }^{11} \mathrm{~B}$ MAS NMR spectra for $\mathrm{Ga}_{1-x} \mathrm{Fe}_{x} \mathrm{BO}_{3}$ powders with $x=0,0.01$ and 0.02 . These spectra reveal a characteristic MAS quadrupolar powder pattern, cf. refs. [8,9]. For ${ }^{11} \mathrm{~B}$ nuclei the shape of MAS NMR spectra is determined by interactions between the nuclear magnetic moments and the applied magnetic field as well as by coupling between the nuclear electric quadrupole moment $Q$ and the electric field gradient (EFG) produced by the surroundings $[8,9]$. The EFG is described by a second rank tensor $V$ with principal components $V_{i i}(i=x, y$ and $z$ ) [8,9]. The symmetry of the EFG is expressed by the parameter $\eta=\left(V_{x x}-V_{y y}\right) / V_{z z}$; in particular, in axial symmetry $V_{x x}=V_{y y}$ and $\eta=0$.

At first, we have simulated MAS NMR spectra with the help of the DMFit program [18]. The best-fit NMR parameters $\delta_{i s o}, C_{Q}, \eta$ and the (Lorentzian) linewidth $\sigma_{\nu}$ for ${ }^{11} \mathrm{~B}$ isotope are given in Table 1 . The corresponding computer-generated MAS NMR spectra are shown in Fig. 1.

In the experimental conditions of the present work, the quadrupole interaction for ${ }^{11} \mathrm{~B}$ isotope is small with respect to the

Table 1

NMR Parameters for ${ }^{11} \mathrm{~B}$ isotope in $\mathrm{Ga}_{1-x} \mathrm{Fe}_{x} \mathrm{BO}_{3}$ obtained with the DMFit program.

\begin{tabular}{llll}
\hline$x$ & 0.00 & 0.01 & 0.02 \\
\hline$\delta_{\text {iso }}, \mathrm{ppm}$ & $24.5 \pm 0.1$ & $24.9 \pm 0.1$ & $27.6 \pm 0.1$ \\
$C_{Q}, \mathrm{MHz}$ & $2.84 \pm 0.02$ & $2.86 \pm 0.02$ & $3.16 \pm 0.02$ \\
$\sigma_{\nu}, \mathrm{kHz}$ & $0.118 \pm 0.01$ & $0.133 \pm 0.01$ & $0.564 \pm 0.01$ \\
$\eta$ & 0 & & \\
\hline
\end{tabular}

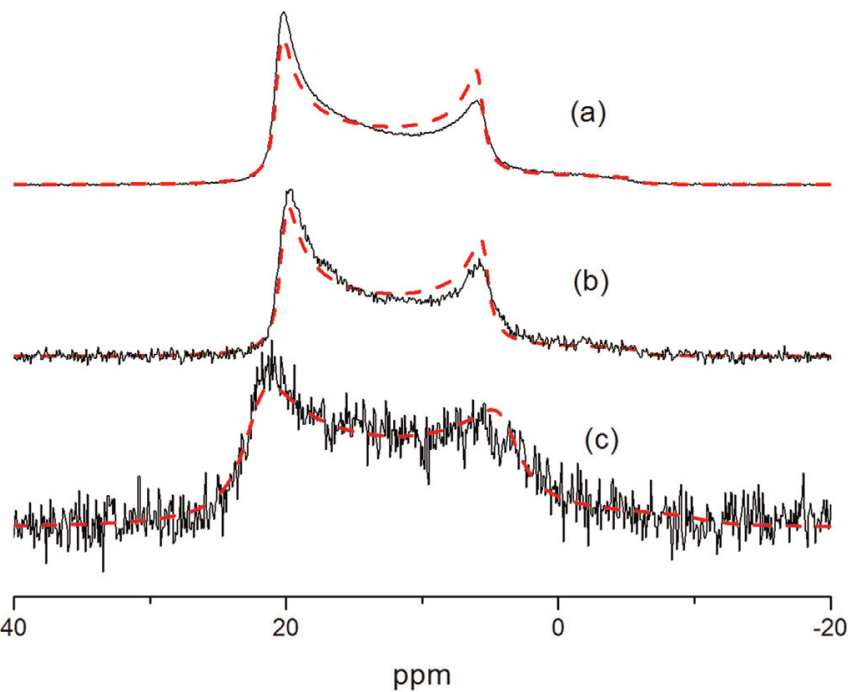

Fig. 1. Experimental (black lines) and computer-generated with the DMFit program (dashed red lines) MAS NMR spectra of ${ }^{11} \mathrm{~B}$ in $\mathrm{Ga}_{1-x} \mathrm{Fe}_{x} \mathrm{BO}_{3}$ with $x=0.00$ (a), 0.01 (b) and 0.02 (c)

Zeeman interaction; therefore, its contribution can be calculated as a perturbation. In the first order, the quadrupole interaction has no effect on the central NMR transition $( \pm 1 / 2 \leftrightarrow \mp 1 / 2)$; only the satellite transitions $( \pm 1 / 2 \leftrightarrow \mp 3 / 2)$ are affected [8,9]. Besides, MAS has no "magic" properties on the second order quadrupole interaction, so, the NMR lines belonging to the central NMR transition will not be averaged. Therefore, in the MAS experiments on powdered samples only the spectrum of the central transition is usually observed $[8,9]$. In the calcite structure of $\mathrm{M}^{3+} \mathrm{BO}_{3}$, the $\mathrm{BO}_{3}$ groups are located in the plane perpendicular to $C_{3}$ axis, so, boron sites have $C_{3}$ local symmetry. The simulation results are consistent with threefold-coordination of boron in $\mathrm{Ga}_{1-x} \mathrm{Fe}_{x} \mathrm{BO}_{3}$ structure [27].

One can see that the DMFit program allows reproducing the main spectra characteristics; however, relative amplitudes and widths of the spectra features cannot be satisfactorily reproduced.

Most obvious mechanisms of broadening of the MAS NMR spectra of ${ }^{11} \mathrm{~B}$ in $\mathrm{Ga}_{1-x} \mathrm{Fe}_{x} \mathrm{BO}_{3}$ with increasing $x$ are the following ones: (i) dipole-dipole coupling between the electron spins of $\mathrm{Fe}^{3+}$ ions and the nuclear spins of the ${ }^{11} \mathrm{~B}$ isotope and (ii) local disorder (statistical site-to-site distribution of atomic positions) caused by iron doping and manifesting itself in distributions of the NMR parameters.

The NMR spectra broadening caused by dipole-dipole interactions would have roughly similar impact on all spectral features. In Fig. 2 we show a series of spectra calculated with different linewidths. One can see that these simulations do not satisfactorily reproduce the shape of the experimental spectra. Therefore, dipole-dipole coupling between paramagnetic nuclei and $\mathrm{Fe}^{3+}$ ions cannot be the leading mechanism of the observed NMR spectra broadening with increasing iron contents.

\subsection{Local disorder and distributions of NMR parameters}

In order to improve the quality of fitting to the experimental MAS NMR spectra, we have assumed the presence of a certain local disorder in the crystals resulting in distributions of the quadrupole parameters for ${ }^{11} \mathrm{~B}$ nuclei in $\mathrm{Ga}_{1-x} \mathrm{Fe}_{x} \mathrm{BO}_{3}$. In this case, different features are expected to broaden to a different extent because of complex and, generally, non-linear relations between the NMR parameters and the resonance frequencies. To handle this situation, we have put forward a simulation code taking into account the quadrupole parameter distribution described by the 


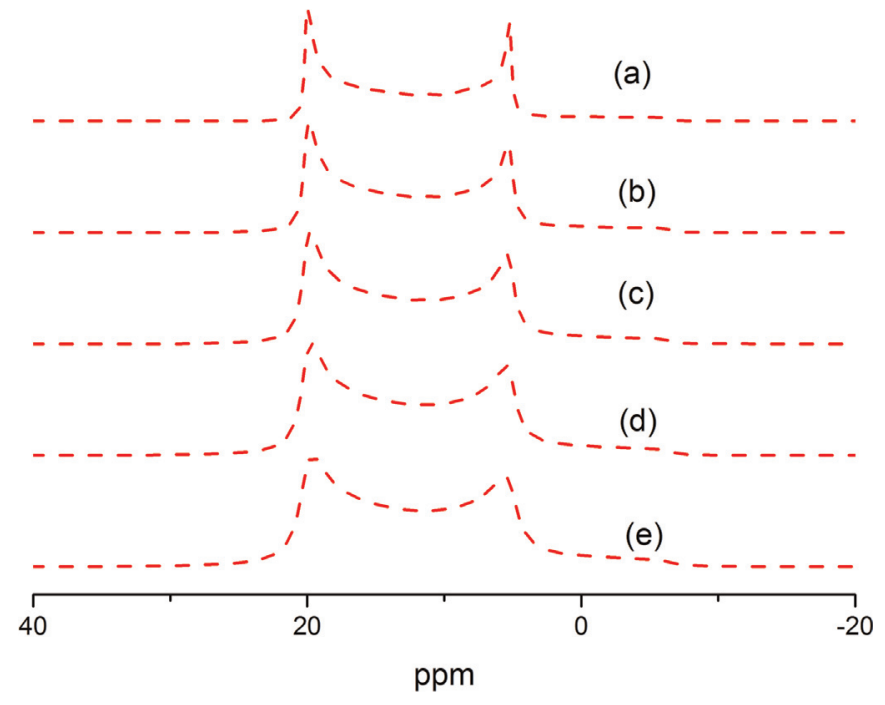

Fig. 2. Series of computer-generated MAS NMR spectra of ${ }^{11} \mathrm{~B}$ with fixed parameters $\delta_{\text {iso }}=24.5 \mathrm{ppm}, \eta=0$ and $\mathrm{C}_{Q}=2.86 \mathrm{MHz}$ for all spectra and different linewidths: $\sigma_{\nu}=0.04$ (a), 0.08 (b), 0.12 (c), 0.16 (d) and $0.20 \mathrm{kHz}$ (e).

Maurer's joint distribution density (JDD) [20] appropriate in the case of randomly disordered structure possessing an intrinsic noncubic local symmetry.

The resonance frequency of the central transition in the MAS experiment up to the second order of the perturbation can be written as $[8,9,28]$

$$
\begin{aligned}
\nu_{r}= & \nu_{L}\left(1+\delta_{i s o}\right)-\frac{\nu_{Q}^{2}}{6 \nu_{L}}\left[I(I+1)-\frac{3}{4}\right] \\
& {\left[\frac{1}{5}\left(1+\frac{\eta^{2}}{3}\right)+A \cos ^{4} \vartheta+B \cos ^{2} \vartheta+C\right] }
\end{aligned}
$$

In Eq. (1), $\nu_{Q}=3 C_{Q} / 2 I(2 I-1)$, and $A, B$ and $C$ are functions of $\eta$ and $\varphi$ given in $[9,28]$. In our case the polar angle $\vartheta$ is defined with respect to the $C_{3}$ axis and the azimuthal angle $\varphi$ is defined with respect to the $C_{2}$ axis (see ref. [2] for the axes definition).

Slightly transforming and amending for a clerical error eq. (10) of ref. [20], the JDD of variables $\Delta$ and $\psi$, related to $C_{Q}$ and $\eta$ as $C_{Q}=\Delta \cos (\pi / 6-\psi)$ and $\eta=\sqrt{3} \tan (\pi / 6-\psi)$, becomes:

$$
\begin{aligned}
P(\Delta, \psi) \propto & \frac{\Delta}{\sigma_{\Delta}} g \cos 3 \psi \\
& \exp \\
& \left\{-\frac{1}{2} \frac{1}{1-\rho_{\Delta \psi}^{2}}\left[\left(\frac{\Delta-\Delta_{0}}{\sigma_{\Delta}}\right)^{2}+\left(\frac{\psi-\psi_{0}}{\sigma_{\psi}}\right)^{2}\right.\right. \\
& \left.\left.-2 \rho_{\Delta \psi} \frac{\left(\Delta-\Delta_{0}\right)\left(\psi-\psi_{0}\right)}{\sigma_{\Delta} \sigma_{\psi}}\right]\right\}
\end{aligned}
$$

where

$g=\frac{\Delta \Delta_{0}}{\sigma_{\Delta}^{2}}-1+\left(\frac{\Delta \Delta_{0}}{\sigma_{\Delta}^{2}}+1\right) e^{-2 \frac{\Delta \Delta 0}{\sigma_{\Delta}^{2}}}$

Here $\Delta_{0}$ and $\psi_{0}$ are mean values, $\sigma_{\Delta}$ and $\sigma_{\psi}$ are distribution widths of the corresponding parameters, and $\rho_{\Delta \psi}$ is a correlation coefficient between $\Delta$ and $\psi$.

The NMR spectra are computed by integrating over distributed values of $\Delta$ and $\psi$, and over different orientations:
$A(\nu) \propto \int_{0}^{2 \pi} \int_{0}^{\pi} \int_{0}^{\pi / 6} \int_{0}^{\infty} P(\Delta, \psi) F\left(\nu-\nu_{r}, \sigma_{\nu}\right) \sin \vartheta d \Delta d \psi d \vartheta d \varphi$

In Eq. (4) $F\left(\nu-\nu_{r}, \sigma_{\nu}\right)$ is an intrinsic lineshape with a linewidth $\sigma_{\nu}$. In the case of line broadening due to distributions of the Hamiltonian parameters, one gets [29]:

$\sigma_{\nu}^{2}=\left(\frac{\partial \nu_{r}}{\partial \Delta}\right)^{2} \sigma_{\Delta}^{2}+\left(\frac{\partial \nu_{r}}{\partial \psi}\right)^{2} \sigma_{\psi}^{2}+2 \frac{\partial \nu_{r}}{\partial \Delta} \frac{\partial \nu_{r}}{\partial \psi} \sigma_{\Delta} \sigma_{\psi} \rho_{\Delta \psi}$

Our computer simulation code ${ }^{1}$ is organized in the following stages.

1. The resonance frequencies, Eq. (1) are calculated by Monte Carlo technique for different orientations of ${ }^{11} \mathrm{~B}$ nuclear spins and different values of the distributed spectroscopic parameters.

2. Each individual resonance is represented by a normalized lineshape function (Lorentzian, Gaussian or other) with a linewidth given in Eq. (5).

3. A MAS NMR spectrum is generated by summing up all individual resonances weighted by the Maurer distribution density, Eqs. (2) and (3), and the $\sin \vartheta$ factor.

The determination of the mean spectroscopic parameters $\Delta_{0}$ and $\psi_{0}$ is straightforward, as far as changing these parameters directly affects the resonance frequencies. On the other hand, distributions of $\Delta$ and $\psi$ mainly affect the NMR spectra shape. The impact of these distributions is illustrated by series of computergenerated spectra shown in Figs. 3-5. As one can see from Fig. 3, increasing of $\sigma_{\Delta}$ leads to a significant broadening of the spectral feature at ca. $5 \mathrm{ppm}$, at the same time, the feature at ca. $20 \mathrm{ppm}$ is much less affected. On the other hand, increasing $\sigma_{\psi}$ results in a significant broadening of the spectra feature at ca. $20 \mathrm{ppm}$, see Fig. 4. Fig. 5 shows the transformation of the computer-generated spectra with varying the correlation coefficient $\rho_{\Delta \psi}$; this parameter particularly affects the shape of the line at ca. $20 \mathrm{ppm}$.

As one can see from Figures 3 to 5, the spectra features are very sensitive to the distribution widths of $\Delta$ and $\psi$ as well as to the correlation coefficient $\rho_{\Delta \psi}$; besides, these characteristics affect the spectra shape in a different manner and extent. Thus, a careful choice of different sets of the fitting parameters allows fine-tuning the shape of a computer-generated spectrum. As a result, very close fittings to the experimental spectra can be obtained.

The best-fit NMR parameters for ${ }^{11} \mathrm{~B}$ nuclei, determined using our laboratory-developed code, are given in Table 2, and the corresponding computer-generated spectra are shown in Fig. 6. Obviously, with this code the quality of fitting to the experimental spectra is considerably improved.

This result provides a strong evidence in support of the idea that the observed NMR spectra broadening in $\mathrm{Ga}_{1-x} \mathrm{Fe}_{x} \mathrm{BO}_{3}$ crystals with increasing iron contents is an increase in local disorder.

In order to further check the consistency of the spectroscopic parameters determined, we have tried to computer simulate a static (measured without rotating the sample) ${ }^{11} \mathrm{~B}$ NMR spectrum in $\mathrm{Ga}_{1-x} \mathrm{Fe}_{x} \mathrm{BO}_{3}$ with $x=0.00$ crystal. The static spectrum corresponding to the central transition has been calculated using Eq. (1) with anisotropic chemical shift added and the A, B and C functions corresponding to the static NMR case, e.g., see ref. [28]. Fig. 7 shows the experimental and computer-generated spectra with the parameters given for this crystal in Table 2. In addition, we were bound to assume an anisotropy of the chemical shift: $\delta_{x}=\delta_{y}=5, \delta_{z}=-10 \mathrm{ppm}$ (this anisotropy is averaged in a MAS NMR [8]). In order to account for the severe broadening of the

\footnotetext{
${ }^{1}$ Available on request from the authors.
} 


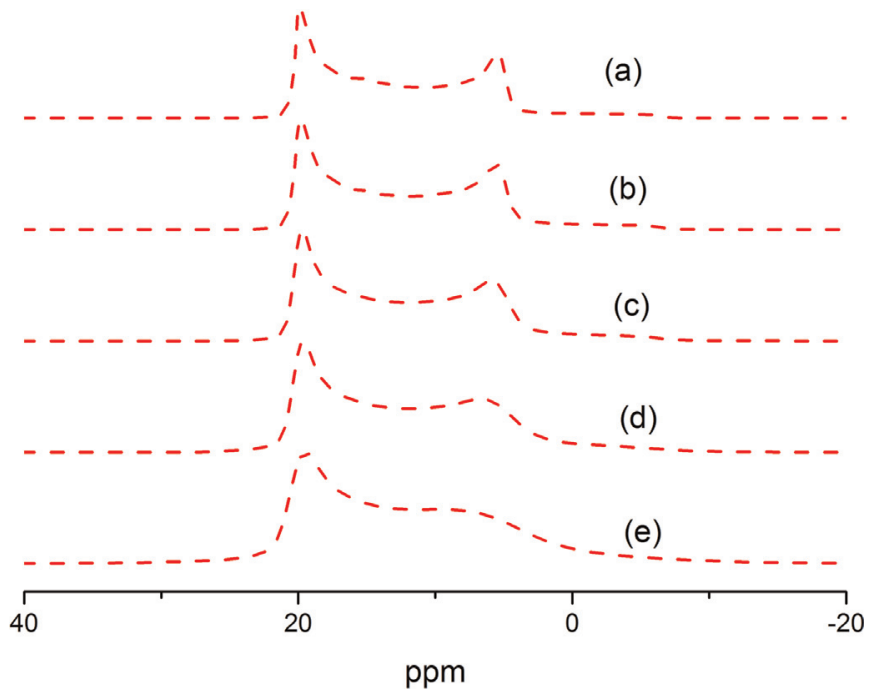

Fig. 3. Series of computer-generated MAS NMR spectra of ${ }^{11} \mathrm{~B}$ with $\psi_{0}=0.523$, $\sigma_{\psi}=0.015$ and $\rho_{\Delta \psi}=0.2$ and different distribution widths of $\Delta: \sigma_{\Delta}=0.01$ (a), 0.02 (b) 0.04 (c, best-fit for $\mathrm{Ga}_{1-x} \mathrm{Fe}_{x} \mathrm{BO}_{3}$ with $x=0.01$ ), 0.08 (d) and $0.16 \mathrm{MHz}$ (c). $\Delta_{0}=2.86 \mathrm{MHz}$ for all spectra.

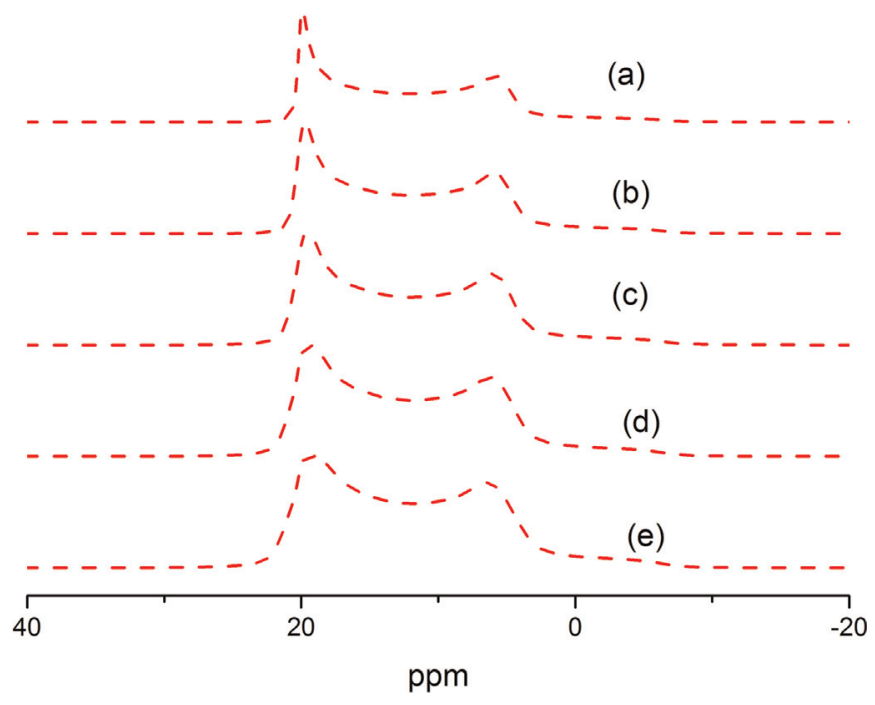

Fig. 4. Computer-generated MAS NMR spectra of ${ }^{11} \mathrm{~B}$ with parameters $\Delta_{0}=2.86 \mathrm{MHz}$ $\sigma_{\Delta}=0.04$ and $\rho_{\Delta \psi}=0.2$ and different distribution widths of $\psi \cdot \sigma_{\psi}=0.005$ (a), 0.015 ((b), best-fit for $\mathrm{Ga}_{1-x} \mathrm{Fe}_{x} \mathrm{BO}_{3}$ with $x=0.01$ ) 0.025 (c), 0.035 (d) and 0.045 (c). $\psi_{0}=0.523$ for all spectra.

static NMR spectrum with respect to the MAS one, a much larger linewidth, $\sigma_{\nu}=3.8 \mathrm{kHz}$ had to be chosen. One can see that, in spite of a somewhat poor signal-to-noise ratio in the experimental spectrum, its main features are convincingly reproduced. This evidence confirms the trustworthiness of the spectroscopic parameters obtained by computer simulating the MAS NMR spectra.

Further experimental work as well as computer simulations of the static NMR spectra in $\mathrm{Ga}_{1-x} \mathrm{Fe}_{x} \mathrm{BO}_{3}$ crystals are in progress.

\section{Conclusions}

The MAS NMR spectroscopy of the ${ }^{11} \mathrm{~B}$ nuclei allows identifying local symmetry and determining local structure for these atoms in $\mathrm{Ga}_{1-x} \mathrm{Fe}_{x} \mathrm{BO}_{3}$ crystals. The simulation parameters obtained for ${ }^{11} \mathrm{~B}$ are consistent with threefold-coordination and $\mathrm{C}_{3}$ intrinsic symmetry for the ${ }^{11} \mathrm{~B}$ nuclei. The behavior of the MAS NMR spectra with the increase in iron contents has been related to variations of

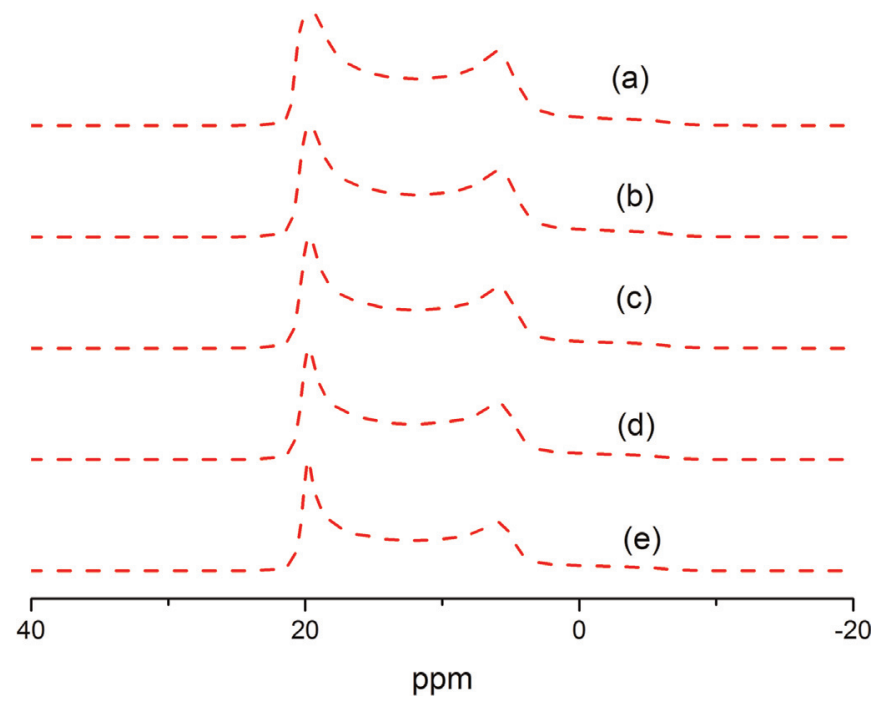

Fig. 5. Computer-generated MAS NMR spectra of ${ }^{11} \mathrm{~B}$ with $\Delta_{0}=2.86 \mathrm{MHz}$, $\sigma_{\Delta}=0.04, \quad \psi_{0}=0.523$ and $\sigma_{\psi}=0.015$ and different correlation coefficients $\rho_{\Delta \psi}$ : -0.8 (a), -0.3 (b), +0.2 ((c), best-fit for $\mathrm{Ga}_{1-x} \mathrm{Fe}_{x} \mathrm{BO}_{3}$ with $\left.x=0.01\right),+0.5$ (d) and $+0.8(\mathrm{e})$.

Table 2

NMR Parameters for ${ }^{11} \mathrm{~B}$ isotope in $\mathrm{Ga}_{1-x} \mathrm{Fe}_{x} \mathrm{BO}_{3}$ obtained with our laboratory-developed code.

\begin{tabular}{llll}
\hline$x$ & 0.00 & 0.01 & 0.02 \\
\hline$\delta_{\text {iso, }}, \mathrm{ppm}$ & $24.7 \pm 0.1$ & $24.5 \pm 0.1$ & $27.6 \pm 0.1$ \\
$\Delta_{0}, \mathrm{MHz}$ & $2.84 \pm 0.02$ & $2.86 \pm 0.02$ & $3.16 \pm 0.02$ \\
$\sigma_{\Delta}, \mathrm{MHz}$ & $0.04 \pm 0.01$ & $0.04 \pm 0.01$ & $0.08 \pm 0.01$ \\
$\psi_{0}$ & 0.510 to 0.523 & & \\
$\sigma_{\psi}$ & $0.011 \pm 0.005$ & $0.015 \pm 0.005$ & $0.027 \pm 0.005$ \\
$\rho_{\Delta \psi}$ & $0.1 \pm 0.1$ & $0.2 \pm 0.1$ & $-0.3 \pm 0.1$ \\
$C_{Q}, \mathrm{MHz}$ & $2.84 \pm 0.02$ & $2.86 \pm 0.02$ & $3.16 \pm 0.02$ \\
$\sigma_{\mathrm{CQ}}$ & $0.04 \pm 0.01$ & $0.04 \pm 0.01$ & $0.08 \pm 0.01$ \\
$\eta_{0}$ & 0.0 to 0.023 & & \\
$\sigma_{\eta}$ & $0.019 \pm 0.009$ & $0.026 \pm 0.009$ & $0.047 \pm 0.009$ \\
$\rho_{\mathrm{CQ} \eta}$ & $-0.1 \pm 0.1$ & $-0.2 \pm 0.1$ & $0.3 \pm 0.1$ \\
\hline
\end{tabular}

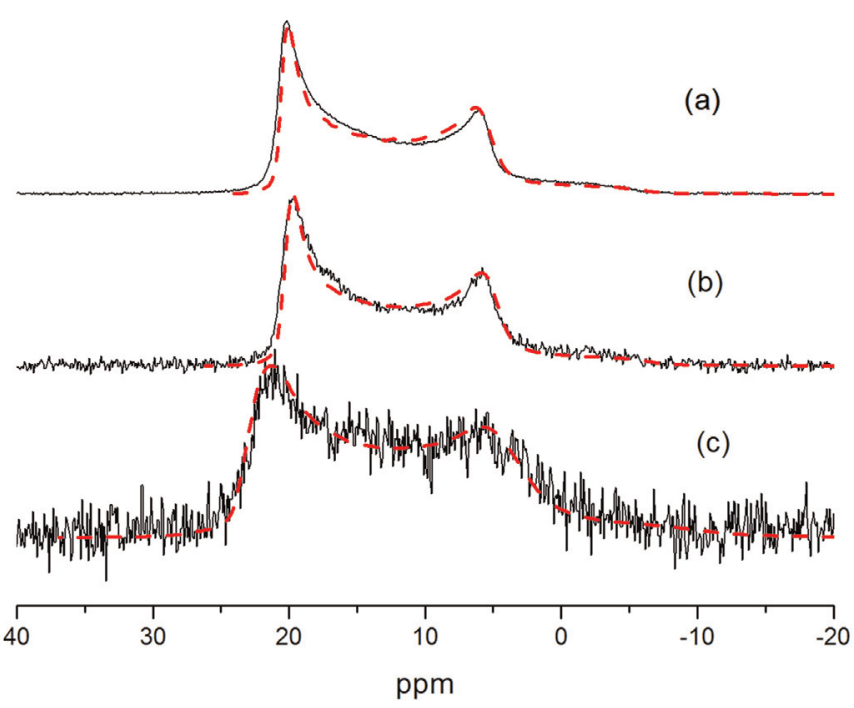

Fig. 6. Experimental (black lines) and computer-generated with our laboratorydeveloped code (dashed red line) MAS NMR spectra of ${ }^{11} \mathrm{~B}$ in $\mathrm{Ga}_{1-x} \mathrm{Fe}_{x} \mathrm{BO}_{3}$ with $x=0.00$ (a), 0.01 (b) and 0.02 (c). 


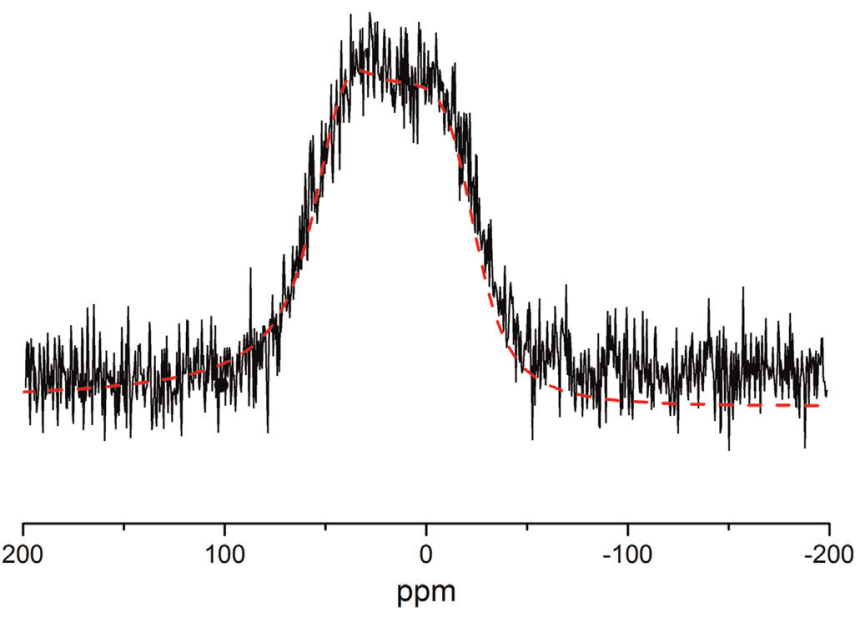

Fig. 7. Experimental (black lines) and computer-generated with our laboratorydeveloped code (dashed red line) static NMR spectrum of ${ }^{11} \mathrm{~B}$ in $\mathrm{Ga}_{1-x} \mathrm{Fe}_{x} \mathrm{BO}_{3}$ with $x=0.00$. See text for the simulation parameters.

quadrupole parameters and chemical shift caused by disorder in the local environment This assumption is corroborated with accurate computer simulations of $\mathrm{Fe}^{3+}$ EPR spectra [21], showing the existence of a certain degree of local disorder in iron-doped gallium borate crystals.

\section{Acknowledgment}

This work was partially supported by RFBR Grant no. 14-4201557

\section{References}

[1] N.V. Kazak, A.D. Balaev, S.G. Ovchinnikov, N.B. Ivanova, V.V. Rudenko, J. Magn. Magn. Mater. 300 (2006) 507.

[2] R. Diehl, W. Jantz, B.I. Nolang, W. Wettling, Current Topics, in: E. Kaldis (Ed.), Materials Science, vol. 11, Elsevier, New-York, 1984, p. 241.
[3] A.L. Kurtzig, R. Wolfe, R.C. LeCraw, J.W. Nielsen, Appl. Phys. Lett. 14 (1969) 350.

[4] W. Jantz, J.R. Sandercock, W. Wettling, J. Phys, C 9 (1976) 2229.

[5] L.V. Velikov, A.S. Prokhorov, E.G. Rudaszevskii, V.N. Seleznev, Sov.Phys. JETP 39 (1974) 909.

[6] M.B. Strugatsky, K.M. Skibinsky, J. Magn. Magn. Mater. 309 (2007) 64.

[7] G. Le Caër, R.A. Brand, J. Phys.: Cond. Matter 10 (1998) 10715.

[8] D. Freude, Quadrupolar nuclei in solid-state nuclear magnetic resonance, in: R. A. Meyers (Ed.), Encyclopedia of Analytical Chemistry, 2000, p. 12188.

[9] D. Freude, J. Haase, in: NMR Basic Principles and Progress, Springer; Berlin, Heidelberg, vol. 29, 3-90 (1993, updated version: August 2014) (http://www. quad-nmr.de).

[10] S.A. Smith, T.O. Levante, B.H. Meier, R.R. Ernst, J. Magn. Res. Ser. A 106 (1994)

[11] M. Bak, T. Rasmussen, N.C. Nielsen, J. Magn. Res 147 (2000) 296.

[12] D. Massiot, H. Thiele, A. Germanus, WinFit, Bruker Report, vol. 140, 1994, p. 43

[13] H.K. Jakobsen, STARS Package, Aarhus University, Denmark,Varian: PaloAlto, CA.

[14] K. Eichele, R.E. Wasylishen, Wsolids NMR Simulation Package. 〈http://ramsey chem.ualberta.ca/software/software.html 2001.

[15] J.P. Amoureux, C. Fernandez, P. Granger, NATO ASI Series C322 (1990) 409.

[16] J.P. Amoureux, C. Fernandez, QUASAR Solid-State NMR Simulation for Quadrupolar Nuclei, Université de Lille, France.

17] T.F. Kemp, M.E. Smith, Solid State NMR 35 (2009) 243.

[18] D. Massiot, F. Fayon, M. Capron, I. King, S. Le Calve, B. Alonso, J.-O. Durand, B. Bujoli, Z. Gan, G. Hoatson, Magn. Res. Chem. 40 (2002) 70.

[19] D.L.A.G. Grimminck, B.J.W. Polman, A.P.M. Kentgens, W. Meerts, J. Magn. Res. 211 (2011).

[20] M. Maurer, Phys. Rev. B 34 (1986) 8996.

[21] K. Seleznyova, M. Strugatsky, S. Yagupov, N. Postivei, A. Artemenko, J. Kliava, Phys. Status Solidi B 251 (2014) 1393.

[22] M.B. Strugatsky, S.V. Yagupov, N.S. Postivey, K.A. Seleznyova, E.T. Milyukova, V. S. Yagupov, Sci. Notes TNU (Simferopol), Ser. Phys. Math. 24 (63) (2011) 169.

[23] B.V. Padlyak, N.A. Sergeev, M. Olszewski, V.T. Adamiv, Ya. V. Burak, Phys. Chem. Glasses: Eur. J. Glass Sci. Technol. B 55 (2014) 25.

[24] A. Abragam, The Principles of Nuclear Magnetism, Clarendon, Oxford, UK, 1961.

[25] E.L. Hahn, Phys. Rev. 80 (1950) 580.

[26] M.E. Smith, E.R.H. van Eck, Prog. NMR Spectrosc. 34 (1999) 159.

[27] S. Kroeker, J.F. Stebbins, Inorg. Chem. 40 (2001) 6239.

[28] D. Müller, Ann. Phys. 39 (1982) 451.

[29] J. Kliava, Phys. Status Solidi B 134 (1986) 411. 